\title{
THE LAST STAGES OF TERRESTRIAL PLANET FORMATION: DYNAMICAL FRICTION AND THE LATE VENEER
}

\author{
Hilke E. Schlichting ${ }^{1,2,4}$, Paul H. Warren ${ }^{1}$, ANd Qing-Zhu Yin $^{3}$ \\ ${ }^{1}$ UCLA, Department of Earth and Space Science, 595 Charles E. Young Drive East, Los Angeles, CA 90095, USA; hilke@ ucla.edu \\ ${ }^{2}$ Department of Astronomy and Astrophysics, California Institute of Technology, MC 130-33, Pasadena, CA 91125, USA \\ ${ }^{3}$ UCD, Department of Geology, One Shields Avenue, Davis, CA 95616, USA \\ Received 2011 November 8; accepted 2012 March 28; published 2012 May 21
}

\begin{abstract}
The final stage of terrestrial planet formation consists of the clean-up of residual planetesimals after the giant impact phase. Dynamically, a residual planetesimal population is needed to damp the high eccentricities and inclinations of the terrestrial planets to circular and coplanar orbits after the giant impact stage. Geochemically, highly siderophile element (HSE) abundance patterns inferred for the terrestrial planets and the Moon suggest that a total of about $0.01 M_{\oplus}$ of chondritic material was delivered as "late veneer" by planetesimals to the terrestrial planets after the end of giant impacts. Here, we combine these two independent lines of evidence for a leftover population of planetesimals and show that: (1) a residual population of small planetesimals containing $0.01 M_{\oplus}$ is able to damp the high eccentricities and inclinations of the terrestrial planets after giant impacts to their observed values. (2) At the same time, this planetesimal population can account for the observed relative amounts of late veneer added to the Earth, Moon, and Mars provided that the majority of the accreted late veneer was delivered by small planetesimals with radii $\lesssim 10 \mathrm{~m}$. These small planetesimal sizes are required to ensure efficient damping of the planetesimal's velocity dispersion by mutual collisions, which in turn ensures sufficiently low relative velocities between the terrestrial planets and the planetesimals such that the planets' accretion cross sections are significantly enhanced by gravitational focusing above their geometric values. Specifically, we find that, in the limit that the relative velocity between the terrestrial planets and the planetesimals is significantly less than the terrestrial planets' escape velocities, gravitational focusing yields a mass accretion ratio of Earth/Mars $\sim\left(\rho_{\oplus} / \rho_{\text {mars }}\right)\left(R_{\oplus} / R_{\text {mars }}\right)^{4} \sim 17$, which agrees well with the mass accretion ratio inferred from HSEs of 12-23. For the Earth-Moon system, we find a mass accretion ratio of $\sim 200$, which, as we show, is consistent with estimates of 150-700 derived from HSE abundances that include the lunar crust as well as the mantle component. We conclude that small residual planetesimals containing about $\sim 1 \%$ of the mass of the Earth could provide the dynamical friction needed to relax the terrestrial planet's eccentricities and inclinations after giant impacts, and also may have been the dominant source for the late veneer added to Earth, Moon, and Mars.
\end{abstract}

Key words: Earth - Moon - planets and satellites: dynamical evolution and stability - planets and satellites: formation

Online-only material: color figures

\section{INTRODUCTION}

Terrestrial planet formation is generally considered to consist of three main stages. The first stage consists of the formation of small planetesimals (e.g., Chiang \& Youdin 2010), the second stage of the coagulation of these small planetesimals into roughly Mars-sized protoplanets (e.g., Ida \& Makino 1993; Weidenschilling et al. 1997), and the third stage is comprised of collisions of a few dozen protoplanets, called giant impacts (e.g., Agnor et al. 1999; Chambers 2001). However, several lines of evidence suggest that a significant amount of mass was left in planetesimals at the end of the giant impact phase in the terrestrial planet region. This, therefore, argues for an additional and final stage of terrestrial planet formation, which consists of the clean-up of the leftover planetesimals. This final stage of terrestrial planet formation is the focus of the present paper.

Evidence for a significant population of planetesimals in the terrestrial planet region at the end of the giant impact phase comes from two different areas of research: Geochemical evidence suggests that the Earth accreted chondritic materials equivalent to about $0.3 \%-0.7 \%$ of its total mass after the end

\footnotetext{
${ }^{4}$ Hubble Fellow.
}

of giant impacts (Walker 2009). The evidence for this "late veneer" comes from highly siderophile elements (HSEs) that are geochemically characterized as having a strong tendency to partition into metal relative to silicates. Hence, the silicate portions of terrestrial planets with rocky cores are expected to be effectively stripped of HSEs after final core segregation. However, a surprisingly high abundance of HSEs has been inferred on the terrestrial planets, which suggests continued planetesimal accretion onto the Earth, Moon, and Mars after core formation (Warren et al. 1999; Walker et al. 2004; Walker 2009). This implies for the Earth-Moon system that a significant amount of mass was accreted after the Moon-forming impact. The geochemical evidence for the late veneer and the estimated amounts of material added to the Earth, Moon, and Mars after the giant impact phase are summarized and discussed in detail in Section 3.

Independently, there is evidence from planet formation models that argues for a population of leftover planetesimals in the terrestrial planet region (see Section 2 for details). This evidence comes from planet formation models that examine the onset of giant impacts (Kenyon \& Bromley 2006; Ford \& Chiang 2007), from simulations of collisions between protoplanets (Benz \& Asphaug 1999) and from the requirement to 
relax the high eccentricities and inclinations of terrestrial planets after giant impacts (Chambers 2001; O'Brien et al. 2006; Raymond et al. 2006; Schlichting \& Sari 2007). These works argue for the existence of a planetesimal population in the terrestrial planet region that still contains a few to $10 \%$ of the mass of the Earth following the epoch of giant impacts.

In this paper, we investigate the accretion of the leftover planetesimals after the end of giant impacts in the inner solar system and show that we can account for the relative amounts of late veneer accreted by the Earth, Moon, and Mars provided that most of it was delivered by small planetesimals. In Section 2, we first estimate the mass left in planetesimals after giant impacts from planet formation models. We summarize the geochemical evidence for a late veneer and estimate the mass of late veneer added to the Earth, Moon, and Mars in Section 3. We calculate the accretion cross section for the Earth, Moon, and Mars for a range of planetesimals velocities and compare our results with the inferred late veneer for these bodies from measured HSE abundances in Section 4. We calculate the typical planetesimal sizes that delivered the late veneer in Section 5 and estimate their accretion timescale in Section 6. Discussion and conclusions follow in Section 7.

\section{LEFTOVER PLANETESIMALS AFTER THE END OF GIANT IMPACTS: EVIDENCE FROM PLANET FORMATION MODELS}

Orbit crossing and giant impacts begin once mutual stirring of the protoplanets can no longer be efficiently damped by small planetesimals. Order of magnitude estimates that balance the stirring rates of the protoplanets with the damping rates due to dynamical friction, which is generated by small planetesimals, find that orbit crossing sets in when $\sigma \sim \Sigma$, where $\Sigma$ and $\sigma$ correspond to the mass surface density in protoplanets and small planetesimals, respectively (Goldreich et al. 2004a). This result has been confirmed by numerical simulations studying the onset of this instability in the terrestrial planet region (Kenyon \& Bromley 2006). Therefore, about 50\% of the total mass still resides in small planetesimals when giant impacts begin. During giant impacts, planetesimal accretion continues and additional "new" planetesimals may be produced as byproducts of giant impacts. Therefore, due to the fact that giant impacts set in when $\sigma \sim \Sigma$ and the possible production of "new" planetesimals in giant impacts themselves, a significant population of planetesimals is expected to still be present after the end of giant impacts.

In addition, leftover planetesimals provide a way to relax the high eccentricities and inclinations of terrestrial planets after giant impacts. $\mathrm{N}$-body simulations predict eccentricities and inclinations for terrestrial planets after giant impacts that are significantly larger than the time averaged values of the terrestrial planets in our solar system (Chambers \& Wetherill 1998; Agnor et al. 1999; Chambers 2001). A population of small planetesimals could provide the dynamical friction that would be needed to damp the eccentricities and inclinations after giant impacts to the observed values of the terrestrial planets. It has already been shown in $\operatorname{direct} N$-body integrations that including a population of less massive planetesimals, in addition to the massive planetary embryos, decreases the final eccentricities and inclinations (Chambers \& Wetherill 1998; Chambers 2001; O'Brien et al. 2006; Raymond et al. 2006). However, due to computational limitations, none of these works were able to include planetesimals small enough such that their collective interactions could be accurately described by dynamical friction. In the limiting case where the terrestrial planets are embedded in a large number of small planetesimals, the damping of the velocity dispersion (i.e., the damping of the eccentricity and inclination) by dynamical friction only depends on the total mass surface density of the planetesimals and is independent of the mass of the individual bodies. Since this limit has not been reached in direct $N$-body simulations, such works underestimate the strength of dynamical friction for a given mass surface density of planetesimals and hence overestimate the mass needed in planetesimals to damp the eccentricities and inclinations. Furthermore, the velocity dispersion of the small planetesimals, $u$, is likely to be damped by mutual planetesimal collisions, which in turn may lead to more effective dynamical friction being exerted on the terrestrial planets. This is because the strength of dynamical friction depends on the relative velocity between the planetesimals and the terrestrial planets, $v_{\text {rel }}$. Collisional damping of the planetesimal velocity dispersion has not been modeled by direct $N$-body simulations, because including of the order of $\sim 10,000$ small planetesimals is still not feasible computationally.

The minimum mass in small leftover planetesimals needed to damp the eccentricities and inclinations of the terrestrial planets to their current values can be estimated by comparing the eccentricity damping timescale to the accretion timescale of the leftover planetesimals. The damping timescale, $t_{\text {damp }}$, due to dynamical friction generated by leftover planetesimals is given by

$$
t_{\mathrm{damp}}=-v \frac{d t}{d v} \sim \frac{\rho R}{\sigma \Omega}\left(\frac{v}{v_{\mathrm{esc}}}\right)^{4},
$$

where $\rho$ is the mean density, $R$ the radius, $v_{\text {esc }}$ is the escape velocity of the terrestrial planets, and $v$ their velocity dispersion (Goldreich et al. 2004b); $\Omega=\sqrt{G M_{\odot} / a^{3}}$ is the Keplerian angular frequency around the Sun, where $M_{\odot}$ is the mass of the Sun and $a$ the semimajor axis. The damping timescale needs to be shorter than the time required for the remaining planetesimals to be accreted by the terrestrial planets. Writing the planetesimal accretion timescale as

$$
t_{\mathrm{acc}}=-\sigma \frac{d t}{d \sigma} \sim \frac{\rho R}{\Sigma \Omega}\left(\frac{v}{v_{\mathrm{esc}}}\right)^{2}
$$

and requiring that $t_{\mathrm{acc}}>t_{\mathrm{damp}}$ yields

$$
\sigma \gtrsim \Sigma\left(\frac{v}{v_{\mathrm{esc}}}\right)^{2}
$$

where $\Sigma$ corresponds here to the mass surface density of the terrestrial planets. Hence, we see from Equation (3) that we can place a lower limit on the mass required in small planetesimals as long as we know the velocity dispersion of the terrestrial planets, $v$, at the end of giant impacts. We assumed in Equations (1)-(3) that $v>u$, where $u$ is the planetesimal velocity dispersion, because small planetesimals are likely to damp their velocities by mutual collisions, ensuring that $u \ll v$. The relative velocity between the planetesimals and terrestrial planets will therefore be dominated by the terrestrial planet's velocity dispersion. We can estimate a minimum value for $v$ by requiring that the velocity dispersion must at least be large enough for neighboring planetary embryos that are undergoing giant impacts to cross their orbits. $N$-body simulations find a typical spacing of planetary embryos of $\sim 8 R_{H}$ (Lissauer 1993), where $R_{H}$ is the Hill radius defined as $R_{H} \equiv a\left(M / 3 M_{\odot}\right)^{1 / 3}$ 
where $M=4 \pi \rho R^{3} / 3$ is the mass of the planetary embryo. This suggests that $v \sim 4 \Omega R_{H} \sim 2.0(R / a)^{1 / 2}\left(M_{\odot} / M\right)^{1 / 6} v_{\text {esc }}$, which evaluates to $v \sim 0.1 v_{\text {esc }}$ for Earth-like terrestrial planets at $1 \mathrm{AU}$. Substituting for $v$ in Equation (3), we find

$$
\sigma \gtrsim 0.01 \Sigma
$$

This order of magnitude estimate suggests that at least $1 \%$ of the total mass needs to still reside in small planetesimals after the giant impact phase in order to damp the terrestrial planets' eccentricities and inclinations to their observed values. For comparison, $N$-body simulations of the giant impact phase, which include smaller planetesimals that are not in the limit in which the collective interactions of the small bodies with the terrestrial planets can be accurately described by dynamical friction, predict eccentricities of $\sim 0.1$ for terrestrial planets after giant impacts (Chambers 2001). These simulations therefore suggest $v \sim 0.27 v_{\text {esc }}$, and hence that $\sigma \gtrsim 0.07 \Sigma$ (Schlichting \& Sari 2007). Since Equation (4) yields only a lower limit on $\sigma$ and since it is only an order of magnitude estimate, we will assume throughout the rest of the paper that at least $1 \%$ of the total mass needs to reside in small planetesimals after the giant impact phase in order to damp the terrestrial planets' eccentricities to their observed values. In addition, we note here that the value derived in Equation (4) could be reduced by a factor of $\sim(a \Omega / 6 v)^{4}$ if gaps form in the planetesimal disk (see Section 6 for details). This is due to the fact that the presence of gaps increases the planetesimal accretion timescale, while not significantly altering the damping timescale.

\section{GEOCHEMICAL EVIDENCE FOR PLANETESIMAL ACCRETION AFTER THE GIANT IMPACT PHASE}

\subsection{Earth}

HSEs are comprised of $\mathrm{Re}, \mathrm{Au}$ and the six platinum-group elements $\mathrm{Os}, \mathrm{Ir}, \mathrm{Ru}, \mathrm{Pt}, \mathrm{Rh}$, and $\mathrm{Pd}$. These elements have very high metal-silicate partition coefficients, which suggests that the silicate portions of rocky bodies with metallic cores should have been stripped of HSEs at the end of core formation. Yet, the relative abundances of these elements in Earth's mantle are broadly similar to chondrites. Absolute concentrations of Ir and Os in Earth's upper mantle are estimated to be $\sim 3-4 \mathrm{ng} \mathrm{g}^{-1}$ (Walker 2009). Although this is more than 100 times lower than the concentrations of these elements found in chondrites, which typically range from $\sim 400$ to $800 \mathrm{ng} \mathrm{g}^{-1}$, these concentrations are higher than low pressure partition coefficients predict (Horan et al. 2003; Walker 2009). Therefore, if the entire mantle harbors HSE abundances similar to the estimate for Earth's upper mantle, then this suggests that, for an Earth mantle mass of $\sim 4.0 \times 10^{27} \mathrm{~g}$, about $1.5-4.0 \times 10^{25} \mathrm{~g}$, or $0.3 \%-0.7 \% M_{\oplus}$, of chondritic material was added to the Earth by late accretion.

\subsection{Mars}

The abundance of HSEs estimated for the Martian mantle is roughly similar to that of Earth's primitive upper mantle (Walker 2009). Warren et al. (1999) estimate the primitive mantle abundance of $\mathrm{Re}, \mathrm{Os}, \mathrm{Ir}$, and $\mathrm{Au}$ for Mars and find that the Martian HSE abundances likely range from 0.34 to 0.66 of the terrestrial values. Assuming that the Martian mantle has a mass of $5.1 \times 10^{26} \mathrm{~g}$, we estimate a mass accretion ratio for Earth/Mars of 12-23, i.e., the Earth accreted 12-23 times more mass as late veneer than Mars. In this estimate, we assumed that the mass accreted by Mars had an HSE composition similar to that accreted by the Earth.

\subsection{Moon}

The total amount of late veneer added to the Earth can be reasonably well estimated from the HSE abundances in its mantle but the situation for the Moon may be somewhat different, because of the rapid formation of a permanent lunar crust by $\sim 100 \mathrm{Myr}$ after the Moon-forming impact, which contrasts with the delayed development of terrestrial cratons (Carlson \& Lugmair 1988; Snyder et al. 2000; Walker et al. 2004). Consequently, the abundances of HSEs in the lunar mantle probe the material accreted before the isolation of the lunar mantle by the crust, whereas the HSE abundances in the lunar crust provide constraints on the accretion of material after crust formation.

Estimates of the HSE content in the lunar mantle have varied considerably from amounts similar to that of Earth's mantle (Ringwood 1992), to amounts about 20 times lower (Warren et al. 1989; Walker et al. 2004; Day et al. 2007; Walker 2009). However, recent works generally tend to favor the lower end of this range (Walker et al. 2004; Day et al. 2007). Using the Walker (2009) estimate that the lunar mantle has a factor of 20 lower HSE concentrations than the terrestrial mantle and assuming a lunar mantle mass of $7 \times 10^{25} \mathrm{~g}$, we find that $1.3-3.5 \times 10^{22} \mathrm{~g}$ of late veneer was added to the lunar mantle, yielding an Earth/Moon mass accretion ratio of about 1100 . However, this estimate ignores an important additional HSE repository: the crust and the upper part of the lunar lithosphere.

Walker et al. (2004) suggested that a significant amount of late veneer may have been deposited into the lunar crust rather than mantle, implying that the majority of the late veneer was accreted by the Moon after the formation of a permanent lunar crust. Impact melt breccias and bulk regolith samples have Os and Ir concentrations averaging 5-15 $\mathrm{ng} \mathrm{g}^{-1}$ (Morgan et al. 1976; Norman et al. 2002). Assuming a lunar crustal mass of $5 \times 10^{24} \mathrm{~g}$ and that the late veneer was delivered by bodies with Os and Ir concentrations similar to chondrites, which typically range from $\sim 400$ to $800 \mathrm{ng} \mathrm{g}^{-1}$, and that Os and Ir concentrations are roughly uniform throughout the crust, no more than $0.3-1.9 \times 10^{23} \mathrm{~g}$ of late veneer was added to the lunar crust (Walker et al. 2004). ${ }^{5}$ This yields an Earth/lunar crust mass accretion ratio ranging from 200 to 700 . In this estimate, we assumed that the Earth and Moon accreted chondritic material with the same Os and Ir abundances such that the actual abundance value cancels in the relative mass accretion ratio. The above estimate for the mass added by late accretion to the Moon assumed, probably somewhat unrealistically, uniform Os and Ir concentrations throughout the lunar crust; below, we derive an independent estimate of the late veneer added to the Moon by examining the ejecta layer and the HSE abundance gradient within it.

The top of the lunar lithosphere is a layer of ejecta accumulated from countless impacts. This layer is commonly termed a megaregolith, but confusion arises because the term megaregolith implies loose debris. Pressure-sensitive sintering (Warren 2011) has probably markedly increased cohesion within the deeper portion of the ejecta layer. For the loose-debris subvolume of the ejecta, a thickness of roughly $2.5 \mathrm{~km}$ has been inferred based on radar constraints on blockiness of ejecta from large craters (Thompson et al. 1979, 2009). But the ejectavolume model of Warren (2011), combined with Frey (2011)'s

\footnotetext{
5 Note: Walker et al. (2004) quote a slightly narrower range of 4-8 $\times 10^{22} \mathrm{~g}$ for the late veneer, because he assumed a slightly narrower range of possible chondritic concentrations of Os and Ir than we have used here, and assumed a lunar crustal mass of $3.7 \times 10^{24} \mathrm{~g}$.
} 
inventory of 90 likely lunar basins with diameters $\gtrsim 300 \mathrm{~km}$, suggests a global mean ejecta accumulation of $\sim 5.8 \mathrm{~km}$. Adding the Procellarum basin to the Frey (2011) inventory would increase the accumulation by $\sim 3.5 \mathrm{~km}$. In Frey's judgment, Procellarum shows no topographic basin, but if not a single impact, this giant region of low elevation and thin crust may reflect a cluster of a few extremely ancient (degraded), large basins not included in Frey (2011). Thus, a reasonable compromise basis for estimating the global mean ejecta accumulation is to add a large fraction, say $1 / 3$, of a Procellarum equivalence of thickness, i.e., $\sim 1.2 \mathrm{~km}$. This leads to a final estimate that the "known" global ejecta accumulation thickness is $\sim 7 \mathrm{~km}$. However, being based on only observable basins $\gtrsim 300 \mathrm{~km}$ in diameter, this estimate is likely low by a significant factor, at least of the order of two.

The global ejecta layer consists mostly of jumbled target (lunar) matter, with a much smaller proportion of impactor/chondritic matter. For estimating the bulk composition of the ejecta layer, the most useful samples are from highly immature regolith. Immature regolith has been thoroughly churned and mixed, but not (at least, not for long) at the very surface of the Moon, and thus is free or nearly free of micrometeorite component and associated enrichment in HSE (McKay et al. 1991; Warren 2004). For the most commonly measured of the HSE, Ir, the average composition of all highland regolith samples is $\sim 12 \mathrm{ng} \mathrm{g}^{-1}$ (Haskin \& Warren 1991). For immature highland regolith, with no micrometeorite component, the average is probably more like $8-10 \mathrm{ng} \mathrm{g}^{-1}$.

The ejecta layer may be only a fraction of the total upperlithosphere component of the Moon's late veneer. Settling of metals in basin-scale impacts has probably produced local HSE concentrations deep within and even below the crust. An ironmeteoritic or ordinary chondritic impactor would contain metal as a major mineral. In large events, where the central, unejected mass of impact melt is slow to cool and solidify, the dense metal component must tend to settle to the very bottom of the impact melt volume. A known example of metal that settled out of a lunar impact melt is the mostly metallic 4.4 gram rock 14286 (Albrecht et al. 1995). Assuming equilibration occurs, only a tiny proportion of metal would suffice to efficiently scavenge the HSE out of a silicate melt-metal system. Diffusion within metal is very rapid. The limiting factor, for the efficiency of HSE scavenging, may be a tendency for the metal components to be so extremely fine grained that they fail to settle. However, in the largest events, the central mass of unejected impact melt is so slow to cool that even its silicate crystallization is believed to entail gravitational differentiation (Warren et al. 1996; Ivanov et al. 2010). The sunken metal probably ended up mostly near the bottom of the "sheet" of central, unejected impact melt, at a depth equivalent to roughly $1 / 10$ the diameter of the transient crater (Warren et al. 1996); i.e., in general, roughly $1 / 20$ the final basin diameter. In other words, the depth at which the metal components predominantly settled was probably of the order of $50 \mathrm{~km}$. It would take a subsequent basin half as large as the original transient crater, if centered at precisely the same point, to begin to excavate the base of the melt "sheet"; i.e., the settled metal. Thus, HSE concentrations found in the megaregolith at the present surface of the Moon may under-represent, possibly by a large factor, the total amount of HSE-rich matter accreted as late veneer.

Although large basin-scale impacts likely played a crucial role in creating and churning the lunar ejecta layer, they may not have contributed significantly to the overall lunar HSE budget of the ejecta layer. This is because, unlike the small and low

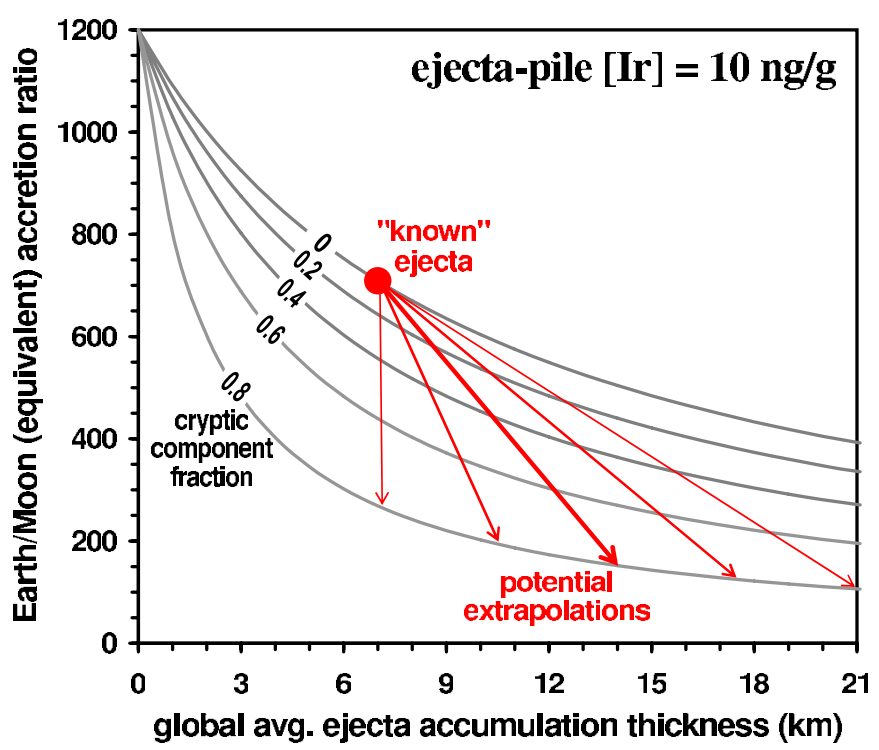

Figure 1. Earth/Moon (equivalent) accretion ratio, shown as a function of assumed mean global thickness of the ejecta layer. The various curves are labeled to indicate different proportion of the cryptic HSE component as a fraction of the total lithospheric HSE component. The cryptic component represents the assumed sequestered HSE component and, in addition, accounts for neveraccreted impactor matter that may have been delivered during the Late Heavy Bombardment. The ejecta layer is assumed to contain $10 \mathrm{ng} \mathrm{g}^{-1} \mathrm{Ir}$.

(A color version of this figure is available in the online journal.)

velocity impactors that collided with the Moon early on and which are the focus of this paper, the largest impactors likely collided late in the lunar history and had large impact velocities, such that only a fraction of the total impact mass was actually accreted by the Moon. For example, the Nice model (Gomes et al. 2005) suggests that the average Late Heavy Bombardment velocity was $21-25 \mathrm{~km} \mathrm{~s}^{-1}$. Under such conditions, at the most common impact angles, only a fraction of the impactor will actually be accreted by the Moon, whereas for the Earth, with its much higher escape velocity, the fraction of impactor matter that fails to accrete is comparatively negligible. Modeling constraints suggest that for rocky impactors the lunar accretion efficiency, integrated over all impact angles, is $0.32-0.16$ for $21-25 \mathrm{~km} \mathrm{~s}^{-1}$ (Artemieva \& Shuvalov 2008; Ong et al. 2010).

We therefore model the total impact mass that has collided with the lunar crust, what may be thought of as the equivalent veneer mass, as a combination of two main components: the ejecta layer itself, with $\sim 10 \mathrm{ng} \mathrm{g}^{-1}$ Ir abundance, and an unobservable, cryptic component. This cryptic component represents the assumed sequestered component of metal that settled out as a result of larger impacts and, in addition, also accounts for never-accreted impactor matter that may have been delivered during the Late Heavy Bombardment. The results in terms of the Earth to Moon (equivalent) accretion ratio, accounting for both the lunar mantle and crust components, are shown in Figure 1, where the various curves correspond to different assumptions for the proportion of the cryptic HSE component as a fraction of the total lithospheric HSE component. Even if we assume that the cryptic component is negligible and that no ejecta accumulation occurred beyond $\sim 7 \mathrm{~km}$ from "known" basins with diameters $\gtrsim 300 \mathrm{~km}$, the Earth/Moon veneer mass ratio is $<700$. This result by itself implies that the total lithospheric HSE component is important, and probably larger than the mantle component, unless the sunken-sequestered component is very small. More realistically, 
the sequestered HSE fraction is probably $\sim 0.5$, but anything between $1 / 5$ and $2 / 3$ seems almost equally plausible, and the total ejecta accumulation is likely at least two times the "known" ejecta volume. The extrapolated Earth/Moon veneer mass ratio is then roughly 300 , assuming $10 \mathrm{ng} \mathrm{g}^{-1} \mathrm{Ir}$ in the ejecta layer. This ratio would be even lower, if inefficient accretion during the Late Heavy Bombardment contributed significantly to the cryptic component. The huge uncertainties in the sequestered HSE fraction and the contribution of inefficient accretion to the cryptic component are permissive of the Earth/Moon veneer mass ratio being conceivably as low as $\sim 150$ or as high as $\sim 700$.

The implied total masses of chondritic-debris additions associated with our various models are perhaps most easily comprehended when expressed in terms of equivalent proportion of chondritic matter within the lunar crust (the "equivalent" qualifier is needed because we assume that a major fraction of the HSE actually became sequestered at the bottoms of the deepest impact melt pools near the base of the crust). Assuming the mass of the crust is $5 \times 10^{24} \mathrm{~g}$, models that suggest Earth/Moon accretion ratios of 600,300 and 150 , imply equivalent proportions of chondritic matter within the crust of about $0.34 \%, 1.0 \%$, and $2.4 \%$ by weight, respectively. ${ }^{6}$ These proportions represent additions to the $1.7 \times 10^{22} \mathrm{~g}$ of chondritic matter inferred to be present in the mantle source region of the lunar (mare) basalts.

In summary, we find that the Earth/Moon mass accretion ratio likely ranges from $\sim 150$ to $\sim 700$. In addition, we note that comparison of the mass accretion estimates for the lunar crust and mantle suggests that most of the late veneer was deposited into the lunar crust rather than mantle, which indicates that most of the late veneer was accreted by the Moon after the formation of a permanent lunar crust (Walker et al. 2004), i.e., about 100 Myr after the Moon-forming impact.

\section{PLANETESIMAL ACCRETION WITH GRAVITATIONAL FOCUSING}

\subsection{The Accretion of Leftover Planetesimals by the Earth and Mars}

If the relative velocity between the planetesimal and the terrestrial planets, $v_{\text {rel }}$, is less than the escape velocity from the terrestrial planets, then the accretion cross sections of the terrestrial planets are enhanced above their geometric values by gravitational focusing. The gravitationally enhanced cross section is given by

$$
A=\pi R^{2}\left[1+\left(\frac{v_{\mathrm{esc}}}{v_{\mathrm{rel}}}\right)^{2}\right]
$$

Using Equation (5), we can write the accretion cross section ratio of Earth/Mars as

$$
\frac{A_{\oplus}}{A_{\mathrm{Mars}}}=\frac{\left(1+\left(v_{\mathrm{esc}}(\oplus) / v_{\mathrm{rel}}\right)^{2}\right) R_{\oplus}^{2}}{\left(1+\left(v_{\mathrm{esc}}(\mathrm{Mars}) / v_{\mathrm{rel}}\right)^{2}\right) R_{\mathrm{Mars}}^{2}},
$$

where the subscripts $\oplus$ and "Mars" label the quantities corresponding to Earth and Mars, respectively. If $v_{\text {rel }} \ll v_{\text {esc }}$, then Equation (6) can be simplified to

$$
\frac{A_{\oplus}}{A_{\text {Mars }}} \sim \frac{\rho_{\oplus}}{\rho_{\text {Mars }}}\left(\frac{R_{\oplus}}{R_{\text {Mars }}}\right)^{4} \sim 17,
$$

\footnotetext{
6 For this illustrative calculation, we assume that all the material delivered to the Earth and Moon was accreted.
}

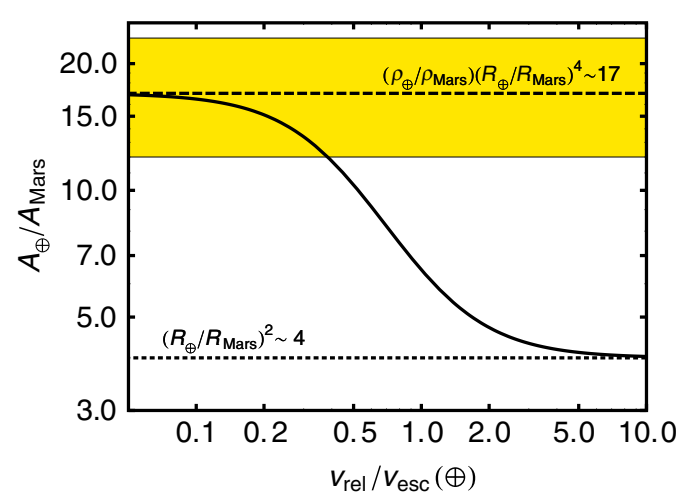

Figure 2. Ratio of the mass accretion rates for the Earth and Mars, $A_{\oplus} / A_{\text {Mars }}$, as a function of $v_{\text {rel }}$ in units of Earth's escape velocity, $v_{\text {esc }}(\oplus)$. The dotted line represents the limit without any gravitational focusing $\left(v_{\mathrm{rel}} \gg v_{\mathrm{esc}}(\oplus)\right)$, in which case $A_{\oplus} / A_{\text {Mars }}=\left(R_{\oplus} / R_{\text {Mars }}\right)^{2} \sim 4$. The dashed line corresponds to the limit with strong gravitational focusing $\left(v_{\text {rel }} \ll v_{\text {esc }}(\oplus)\right)$, in which case $A_{\oplus} / A_{\text {Mars }}=\left(\rho_{\oplus} / \rho_{\text {Mars }}\right)\left(R_{\oplus} / R_{\text {Mars }}\right)^{4} \sim 17$. The yellow shaded region shows the range of Earth/Mars mass accretion ratios that are consistent with the inferred late veneer for the Earth and Mars.

(A color version of this figure is available in the online journal.)

where $\rho_{\oplus}$ and $\rho_{\text {Mars }}$ correspond to the mean density of the Earth and Mars, respectively. This implies that the accretion ratio between the Earth and Mars that has been estimated from the HSE abundances $\left(A_{\oplus} / A_{\text {Mars }} \sim 12-23\right.$, see Section 3$)$ agrees very well with the expected mass accretion ratio between the Earth and Mars, if the accretion cross sections of the Earth and Mars were significantly enhanced by gravitational focusing. For comparison, if $v_{\text {rel }}$ had been larger than the escape velocities of Earth and Mars such that gravitational focusing becomes irrelevant, then the ratio of the accretion cross section Earth/Mars is simply given by $\left(R_{\oplus} / R_{\text {Mars }}\right)^{2} \sim 4$. This value is significantly lower than the mass ratio of the late veneer that has been estimated from the terrestrial and Martian abundances of HSEs (see Figure 2). We therefore conclude that we can account for the relative amounts of late veneer accreted by the Earth and Mars, if it was delivered concurrently by a population of planetesimals with a velocity dispersion small enough such that $v_{\text {rel }} \ll v_{\text {esc }}$.

\subsection{The Accretion of Leftover Planetesimals by the Earth and the Moon}

We can extend the above argument to the Earth and the Moon to get a rough estimate for the Earth/Moon accretion ratio. However, using the expression for gravitational focusing from Equation (6) is, strictly speaking, only valid for isolated bodies and is therefore only a rough approximation for the Earth/Moon accretion ratio. Estimating the Earth/Moon accretion ratio from Equation (6) we have

$$
\frac{A_{\oplus}}{A_{\text {Moon }}} \sim \frac{\rho_{\oplus}}{\rho_{\text {Moon }}}\left(\frac{R_{\oplus}}{R_{\text {Moon }}}\right)^{4} \sim 300 .
$$

This implies that in the limit in which we can treat the Earth and Moon as isolated bodies, i.e., for very large Earth-Moon separations, the Earth should have accreted about 300 times more mass as late veneer compared to the Moon (see Figure 3).

Bandermann \& Singer (1973) derived analytically the ratio of the Earth/Moon accretion cross section. Assuming an isotropic planetesimal velocity distribution far from the Earth and 


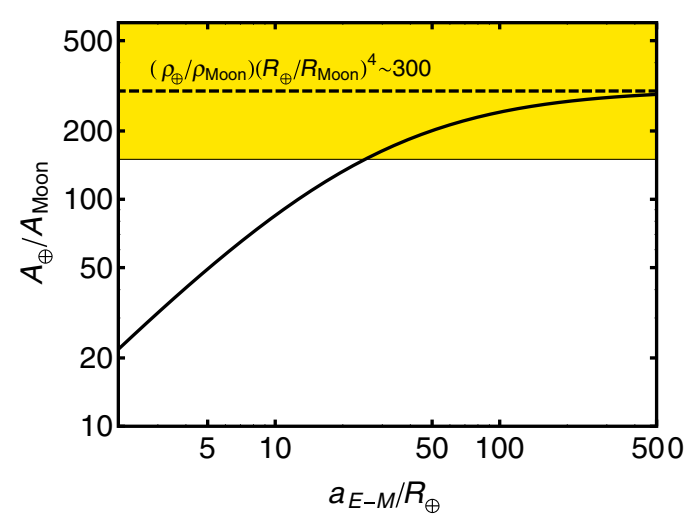

Figure 3. Ratio of the mass accretion rates for the Earth and Moon, $A_{\oplus} / A_{M o o n}$, as a function of the Earth-Moon distance in Earth radii, $a_{\mathrm{E}-\mathrm{M}} / R_{\oplus}$, with strong gravitational focusing (i.e., $v_{\text {rel }} \ll v_{\text {esc }}$ ). The solid line is the analytic result from Bandermann \& Singer (1973) given in Equation (9). The dashed line represents our estimate for the ratio of the Earth/Moon accretion cross section from Equation (8), which is valid for large Earth-Moon separations when the Earth and the Moon can be well approximated as isolated bodies. The yellow shaded region shows the range of Earth/Moon mass accretion ratios that are consistent with the inferred late veneer added to the Earth and Moon based on HSE observations.

(A color version of this figure is available in the online journal.)

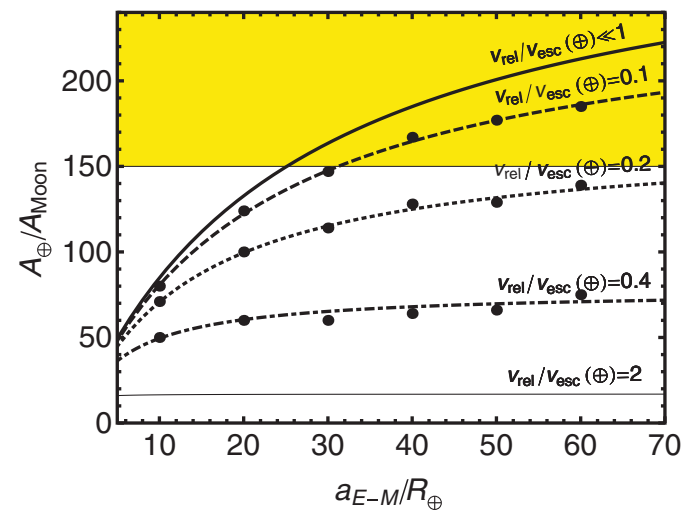

Figure 4. Ratio of the mass accretion rates for the Earth and Moon, $A_{\oplus} / A_{M o o n}$, as a function of the Earth-Moon distance in Earth radii, $a_{\mathrm{E}-\mathrm{M}} / R_{\oplus}$, for various relative velocities, $v_{\text {rel }}$, from Equation (9) (Bandermann \& Singer 1973). For comparison, we also show the results from our numerical simulations (shown as points) in which we integrate the trajectories of the planetesimals in the Earth-Moon system directly. The yellow shaded region shows the range of Earth/Moon mass accretion ratios that are consistent with the inferred terrestrial and lunar late veneer based on HSE observations.

(A color version of this figure is available in the online journal.)

neglecting Earth's shadow they find

$$
\frac{A_{\oplus}}{A_{\mathrm{Moon}}} \geqslant \frac{1+\left(v_{\mathrm{rel}} / v_{\mathrm{esc}}(\oplus)\right)^{2}}{\frac{7}{6}\left(R_{\oplus} / a_{\mathrm{E}-\mathrm{M}}\right)+0.045+\left(v_{\mathrm{rel}} / v_{\mathrm{esc}}(\oplus)\right)^{2}}\left(\frac{R_{\oplus}}{R_{\mathrm{Moon}}}\right)^{2},
$$

where $a_{\mathrm{E}-\mathrm{M}}$ is the Earth-Moon separation. Equation (9) is a lower limit to $A_{\oplus} / A_{\text {Moon }}$ because the effect of Earth's shadow was neglected in deriving the accretion cross section for the Moon, i.e., it neglects lunar impactors that would have collided with Earth first. We confirmed this analytic result for the Earth/Moon accretion cross section by direct numerical integrations of planetesimal trajectories in the Earth-Moon system (see Figure 4).

Figure 3 shows the Earth/Moon accretion ratio as a function of the Earth-Moon separation for $v_{\text {rel }} \ll v_{\text {esc }}$. As expected, the Earth-Moon accretion cross section approaches the ratio for isolated bodies at large Earth-Moon separations. At the current Earth-Moon separation, which corresponds to about $60 R_{\oplus}$, the accretion ratio is $\sim 200$. The Earth/Moon accretion ratio increases with increasing Earth-Moon separation approaching the limit derived in Equation (8) for isolated bodies, this behavior remains unchanged as long as gravitational focusing plays a significant role in enhancing the accretion cross section above the geometric value (see Figure 4). This result may seem surprising at first, because Earth's gravitational field accelerates the incoming planetesimals such that the Moon's gravitational focusing is reduced. However, Earth's gravitational field also focuses the incoming planetesimals such that the Moon intercepts a larger number of planetesimals than it would have otherwise. As a result, the Earth/Moon accretion cross section decreases with decreasing Earth-Moon separation, which implies that it was lower in the past before the Moon evolved tidally outward to its current location. The tidal evolution timescale for the Moon to evolve from an initial separation of a few times Earth's radius to a separation $a_{\mathrm{E}-\mathrm{M}} \gg R_{\oplus}$ is

$$
\begin{aligned}
t_{\text {tidal }}= & \frac{2}{39} \frac{Q}{k} \frac{M_{\oplus}}{M_{\text {Moon }}}\left(\frac{a_{\mathrm{E}-\mathrm{M}}}{R_{\oplus}}\right)^{5}\left(\frac{G M_{\oplus}}{a_{\mathrm{E}-\mathrm{M}}^{3}}\right)^{-1 / 2} \\
& \sim 1.3 \times 10^{4} \frac{Q / 12}{k / 0.299}\left(\frac{a_{\mathrm{E}-\mathrm{M}}}{10 R_{\oplus}}\right)^{6.5} \mathrm{yr},
\end{aligned}
$$

where $k$ and $Q$ are the tidal dissipation function and the tidal Love number of the Earth, respectively. From Equation (10), we see that the initial tidal evolution was very fast such that the Earth-Moon system did not spend a significant amount of time at small Earth-Moon separations and evolved to $a_{\mathrm{E}-\mathrm{M}} \gtrsim 40 R_{\oplus}$ within about $110 \mathrm{Myr}$ of the Moon forming impact. This estimate assumes that $Q \sim 12$ and that its value did not significantly change throughout the tidal evolution. In Section 6, we calculate a planetesimal accretion timescale of $\sim 170 \mathrm{Myr}$, which suggests that most of the planetesimals were accreted after the Earth-Moon system evolved to separations of $\gtrsim 40 R_{\oplus}$. We therefore conclude that the relative amounts of late veneer added to the Moon and Earth, as inferred from their HSE abundances, are consistent with the accretion of small planetesimals with a velocity dispersion of $u \lesssim v_{\text {rel }} \sim 0.1 v_{\text {esc }}(\oplus)$. In contrast, if $v_{\text {rel }} \gg v_{\text {esc }}$, i.e., in the limit without any gravitational focusing, then the Earth/Moon accretion ratio is independent of the Earth-Moon separation and is simply given by the ratio of the geometric cross sections $\left(R_{\oplus} / R_{\text {Moon }}\right)^{2} \sim 14$ (see Figure 4 ), which is inconsistent with the Earth/Moon mass accretion ratio inferred from HSEs of $150-700$.

\section{PLANETESIMAL SIZES}

We have shown in Section 4 that the relative amounts of late veneer delivered to the Earth, Moon, and Mars can be explained by the accretion of small planetesimals after giant impacts, provided that gravitational focusing played a significant role in increasing the accretion cross section above the geometric value. Since gravitational focusing only acts when $v_{\text {rel }} \ll v_{\text {esc }}$, we conclude that the velocity dispersion of the planetesimals was comparable to or less than the velocity dispersion of the terrestrial planets, i.e., $u \lesssim v$, such that $v_{\text {rel }} \sim v$. Furthermore, we can use our estimate from Section 2 that $v \sim 0.1 v_{\text {esc }}$ to place an upper limit on the typical planetesimal size as follows.

The velocity dispersion of the planetesimals is stirred gravitationally by the terrestrial planets and damped by mutual 
planetesimal collisions such that

$$
\frac{1}{u} \frac{d u}{d t} \sim \frac{\Sigma \Omega}{\rho R}\left(\frac{v_{\mathrm{esc}}}{v_{\mathrm{rel}}}\right)^{2}\left(\frac{v_{\mathrm{esc}}}{u}\right)^{2}-\frac{\sigma \Omega}{\rho_{s} s},
$$

where $s$ and $\rho_{s}$ are the radius and density of the planetesimals and $u \leqslant v_{\text {rel }}$. Balancing the stirring and the damping rates yields

$$
s \sim \frac{\sigma \rho}{\Sigma \rho_{s}}\left(\frac{v_{\mathrm{rel}}}{v_{\mathrm{esc}}}\right)^{2}\left(\frac{u}{v_{\mathrm{esc}}}\right)^{2} R
$$

Evaluating Equation (12) for $\sigma / \Sigma \sim 1 \%$ (see discussion in Section 2), substituting for $v_{\text {rel }} \sim v \sim 0.1 v_{\text {esc }}$ and using $u \sim v$, we have

$$
s \sim 10 \mathrm{~m}
$$

where we assumed $\rho_{s} \sim 3 \mathrm{~g} \mathrm{~cm}^{-3}, R \sim R_{\oplus}$ and $\rho \sim \rho_{\oplus}$. This implies that $u \lesssim v \sim 0.1 v_{\text {esc }}(\oplus)$ as long as the typical planetesimals, that damped the eccentricities and inclinations of the terrestrial planets and that delivered the late veneer, were smaller than about $10 \mathrm{~m}$ in size. We assumed when evaluating Equation (13) that $u \sim v$. The actual planetesimal sizes could therefore be smaller than estimated in Equation (13), which would imply that $u<v$. The corresponding optical depth of such a planetesimal population is $\tau \sim \sigma / \rho_{s} s$, which implies $\tau \gtrsim 2.5 \times 10^{-5}$. We also note here that recent work by Weidenschilling (2011) showed that the size distribution of the asteroid belt can be reproduced by coagulation from an initial population of planetesimals as long as they have sizes $\lesssim 100 \mathrm{~m}$, providing independent support for a planetesimal population in the inner solar system that was smaller than about $100 \mathrm{~m}$ in size.

If typical planetesimal sizes would have exceeded about $10 \mathrm{~m}$, then $u>v$ such that $v_{\text {rel }} \sim u>0.1 v_{\text {esc }}$, which would imply weaker or no gravitational focusing, making it hard to reconcile the resulting Earth/Moon and Earth/Mars accretion ratios with the relative quantities of late veneer delivered to these bodies. Furthermore, if $u>v \sim 0.1 v_{\text {esc }}$, then this would imply that more mass than we estimated in Section 2 must have been residing in small planetesimals at the end of giant impacts in order to damp the eccentricities and inclinations of the terrestrial planets.

\section{GAP FORMATION AND ACCRETION TIMESCALES}

Gaps were not important before the end of the giant impact phase, because the radial separation of protoplanets was only a few times larger than the widths of their Hill radii. But at the end of giant impacts, when the terrestrial planets achieved large-scale orbital stability, their orbital separation was much larger than their Hill radii and gaps likely formed around their orbits (Goldreich et al. 2004a). Gaps increase the clean-up timescale of the leftover planetesimals, because accretion onto the protoplanets can now only proceed from the gap edges. Following Goldreich et al. (2004a), the rate at which a terrestrial planet accretes small planetesimals from gap edges located a distance $\mathrm{x}$ from the planets' semimajor axis is

$$
\frac{1}{M} \frac{d M}{d t} \sim \frac{\sigma_{0} \Omega}{\rho R}\left(\frac{2 x}{\Delta a}\right)^{4}\left(\frac{v_{\mathrm{esc}}}{v_{\mathrm{rel}}}\right)^{2},
$$

where $\Delta a$ is the distance between neighboring planets. The gap surface density of the small bodies follows a power law such that the mass surface density at the gap edges is given by $\sigma=$ $\sigma_{0}(2 x / \Delta a)^{4}$. Writing $t_{\mathrm{acc}}=-\sigma_{0} \frac{d t}{d \sigma_{0}} \sim 2 \pi \sigma_{0} a \Delta a(d M / d t)^{-1}$ yields

$$
t_{\mathrm{acc}}=-\sigma_{0} \frac{d t}{d \sigma_{0}} \sim \frac{\rho R}{\Sigma \Omega}\left(\frac{v_{\mathrm{rel}}}{v_{\mathrm{esc}}}\right)^{2}\left(\frac{\Delta a}{2 x}\right)^{4},
$$

where $\Sigma=M /(2 \pi a \Delta a)$. The spacing between the terrestrial planets is $\Delta a \sim a / 3$ and, since $v>u, x$ will be roughly given by the radial excursions of the planet, which is $\sim a e$. This yields a clean-up timescale of

$$
t_{\mathrm{acc}}=-\sigma_{0} \frac{d t}{d \sigma_{0}} \sim \frac{\rho R}{\Sigma \Omega}\left(\frac{v_{\mathrm{rel}}}{v_{\mathrm{esc}}}\right)^{2}\left(\frac{1}{6 e}\right)^{4} \sim 170 \mathrm{Myr}
$$

when evaluated at $1 \mathrm{AU}$ for $R=R_{\oplus}, M=M_{\oplus}$, and $v_{\text {rel }} \sim e a \Omega \sim 0.1 v_{\text {esc }}$. This implies that most of the planetesimals were accreted after the formation of a permanent lunar crust. This result is consistent with the fact that the majority of the late veneer seems to reside in the lunar crust rather than the mantle (see Section 3 for details). Because the planetesimal accretion timescale is long compared to the tidal evolution timescale of the Earth-Moon system (see Equation (10) in Section 4), this implies that the Earth-Moon separation was already $\gtrsim 40 R_{\oplus}$ when most of the late veneer was delivered to the Earth-Moon system.

\section{DISCUSSION AND CONCLUSIONS}

The abundances of HSEs suggest that a total of about $0.01 M_{\oplus}$ was delivered as "late veneer" by planetesimals to the terrestrial planets after the giant impact phase. We showed here that small residual planetesimals, with radii of $\lesssim 10 \mathrm{~m}$, containing about $\sim 1 \%$ of the mass of the Earth can provide the dynamical friction needed to relax the terrestrial planets' eccentricities and inclinations after giant impacts and simultaneously account for the relative and absolute amounts of late veneer added to the Earth, Moon, and Mars. Small planetesimal sizes are required to ensure efficient damping of the planetesimal's velocity dispersion by mutual collisions, which in turn ensures sufficiently low relative velocities between the terrestrial planets and the planetesimals such that the planets' accretion cross sections are significantly enhanced by gravitational focusing above their geometric values. Specifically, we find that, if $v_{\text {rel }} \ll v_{\text {esc }}$, then gravitational focusing yields a mass accretion ratio of Earth/Mars $\sim\left(\rho_{\oplus} / \rho_{\text {mars }}\right)\left(R_{\oplus} / R_{\text {mars }}\right)^{4} \sim 17$, which agrees well with the mass accretion ratio inferred from HSEs of 12-23. For the Earth-Moon system, we find a mass accretion ratio of $\sim 200$, which, as we show in Section 3, is consistent with estimates of 150-700 derived from HSE abundances that include the lunar crust as well as the mantle component. Furthermore, we find that the higher abundance of siderophilic elements in the lunar crust compared to the lunar mantle is consistent with the idea that most of the late veneer was delivered by small planetesimals. This is because, if the residual planetesimals were indeed small, gaps will likely form around the terrestrial planets, which will prolong the planetesimal accretion timescale such that most of the late veneer is added to the lunar crust after the lunar mantle was isolated by the formation of a permanent crust. We note here that, although we suggest that the majority of the accreted late veneer was delivered by small planetesimals, larger planetesimals were certainly residing among the small planetesimal population and must have played a crucial role in the mixing and settling of HSEs in the lunar crust.

Bottke et al. (2010) suggested recently that most of the late veneer may have been delivered by a few very large planetesimals, with the largest terrestrial impactor exceeding more than 
$1000 \mathrm{~km}$ in radius. Delivering the majority of the late veneer by one or two very large bodies may explain stochastically a large mass accretion ratio between the Earth and Moon. However, whereas small body accretion can account for the relative quantities of late veneer inferred from HSE abundances for the Earth/Moon and Earth/Mars simultaneously, it would remain a coincidence in a stochastic accretion scenario. Furthermore, if a small number of $1000 \mathrm{~km}$ sized planetesimals were indeed responsible for the late veneer, then these planetesimals cannot have damped the eccentricities and inclinations of the terrestrial planets after giant impacts. This is because, if most of the planetesimal mass resided in such large bodies, then they would have to have a significantly higher velocity dispersion, because mutual planetesimal collisions that damp their velocities are significantly less frequent for larger planetesimals compared to small ones (see Section 5). In this case, $v_{\text {rel }}$ would be determined by the velocity dispersion of these large planetesimals, which in turn implies that significantly more than $1 \%$ of the total mass would be required in large planetesimals to damp the eccentricities and inclinations of the terrestrial planets (see Section 2). This, however, would be inconsistent with the $\lesssim 0.01 M_{\oplus}$ of chondritic material delivered as late veneer to the Earth, Moon, and Mars. In principle, a population of planetesimals made of primarily silicates with extremely low HSE abundances could have provided the required dynamical friction. However, the relative abundances of the different HSEs in the terrestrial planets and the Moon are consistent with chondritic material and hence favor the idea that they were delivered by small, undifferentiated planetesimals with chondritic composition.

Finally, as we have shown in Section 3, the Earth/Moon impact ratio likely falls in the range of 150-700, once the HSE deposited into the lunar crust and the upper part of the lunar lithosphere are accounted for (see also Walker et al. 2004). If the ratio of the late veneer accreted by the Earth and Moon falls at the lower end of this range, then it is consistent with small body accretion. However, if it can be conclusively shown that the Earth/Moon mass accretion ratio lies at the upper end of this range, then it cannot be explained by the small body accretion discussed here. In this case, it could instead be either due to a small number of stochastic events that delivered most of the late veneer (Bottke et al. 2010) or due to a smaller retention fraction of the material delivered to the Moon compared to the Earth as might be expected for impact velocities significantly exceeding the lunar escape velocity.

We thank David Jewitt and our two referees, John Chambers and Richard Walker, for valuable comments that helped to improve this manuscript. For H.S., support for this work was provided by NASA through the Hubble Fellowship Grant HST-HF-51281.01-A awarded by the Space Telescope Science Institute, which is operated by the Association of Universities for Research in Astronomy, Inc., for NASA, under contact NAS 5-26555. P.W. acknowledges support from the NASA grant NNX09 AE31 G. Q.Z.Y. acknowledges the NASA Cosmochemistry (NNX11AJ51G) and Origins of Solar Systems (NNX09AC93G) grants for support.

\section{REFERENCES}

Agnor, C. B., Canup, R. M., \& Levison, H. F. 1999, Icarus, 142, 219

Albrecht, A., Herzog, G. F., Klein, J., et al. 1995, in Lunar and Planetary Institute Science Conference Abstracts, 13

Artemieva, N. A., \& Shuvalov, V. V. 2008, Sol. Syst. Res., 42, 329

Bandermann, L. W., \& Singer, S. F. 1973, Icarus, 19, 108

Benz, W., \& Asphaug, E. 1999, Icarus, 142, 5

Bottke, W. F., Walker, R. J., Day, J. M. D., Nesvorny, D., \& Elkins-Tanton, L. 2010, Science, 330, 1527

Carlson, R. W., \& Lugmair, G. W. 1988, Earth Planet. Sci. Lett., 90, 119

Chambers, J. E. 2001, Icarus, 152, 205

Chambers, J. E., \& Wetherill, G. W. 1998, Icarus, 136, 304

Chiang, E., \& Youdin, A. N. 2010, Annu. Rev. Earth Planet. Sci., 38, 493

Day, J. M. D., Pearson, D. G., \& Taylor, L. A. 2007, Science, 315, 217

Ford, E. B., \& Chiang, E. I. 2007, ApJ, 661, 602

Frey, H. 2011, in Recent Advances and Current Research Issues in Lunar Stratigraphy, ed. W. A. Ambrose \& D. A. Williams (Boulder, CO: Geological Society of America), 53

Goldreich, P., Lithwick, Y., \& Sari, R. 2004a, ApJ, 614, 497

Goldreich, P., Lithwick, Y., \& Sari, R. 2004b, ARA\&A, 42, 549

Gomes, R., Levison, H. F., Tsiganis, K., \& Morbidelli, A. 2005, Nature, 435, 466

Haskin, L. A., \& Warren, P. H. 1991, in Lunar Sourcebook, A User's Guide to the Moon, ed. G. Heiken, D. Vaniman, \& B. M. French (Cambridge, MA: Cambridge University Press), 357

Horan, M., Walker, R., Morgan, J. W., \& Grossman, J. N. 2003, Chem. Geol., 196,5

Ida, S., \& Makino, J. 1993, Icarus, 106, 210

Ivanov, B. A., Melosh, H. J., \& Pierazzo, E. 2010, in Large Meteorite Impacts and Planetary Evolution IV, ed. R. L. Gibson \& W. U. Reimold (Boulder, CO: Geological Society of America), 29

Kenyon, S. J., \& Bromley, B. C. 2006, AJ, 131, 1837

Lissauer, J. J. 1993, ARA\&A, 31, 129

McKay, D. S., Heiken, G., Basu, A., et al. 1991, in Lunar Sourcebook, A User's Guide to the Moon, ed. G. Heiken, D. Vaniman, \& B. M. French (Cambridge, MA: Cambridge University Press), 285

Morgan, J. W., Gros, J., Takahashi, H., \& Hertogen, J. 1976, in Lunar and Planetary Science Conference Proceedings, ed. R. B. Merrill (New York: Pergamon Press Inc.), 2189

Norman, M. D., Bennett, V. C., \& Ryder, G. 2002, Earth Planet. Sci. Lett., 202, 217

O’Brien, D. P., Morbidelli, A., \& Levison, H. F. 2006, Icarus, 184, 39

Ong, L., Asphaug, E. I., Korycansky, D., \& Coker, R. F. 2010, Icarus, 207, 278

Raymond, S. N., Quinn, T., \& Lunine, J. I. 2006, Icarus, 183, 265

Ringwood, A. E. 1992, Earth Planet. Sci. Lett., 111, 537

Schlichting, H. E., \& Sari, R. 2007, ApJ, 658, 593

Snyder, G. A., Borg, L. E., Nyquist, L. E., \& Taylor, L. A. 2000, in Origin of the Earth and Moon, ed. R. M. Canup \& K. Righter et al. (Tucson, AZ: Univ. Arizona Press), 361

Thompson, T. W., Campbell, B. A., Ghent, R. R., \& Hawke, B. R. 2009, Geology, 37,655

Thompson, T. W., Roberts, W. J., Hartmann, W. K., Shorthill, R. W., \& Zisk, S. H. 1979, Moon Planets, 21, 319

Walker, R. J. 2009, Chem. Erde-Geochem., 69, 101

Walker, R. J., Horan, M. F., Shearer, C. K., \& Papike, J. J. 2004, Earth Planet. Sci. Lett., 224, 399

Warren, P. H. 2004, in Treatise on Geochemistry, Volume 1, Meteorites, Comets, and Planets, ed. A. M. Davis (Amsterdam: Elsevier), 559

Warren, P. H. 2011, Meteorit. Planet. Sci., 46, 53

Warren, P. H., Claeys, P., \& Cedillo-Pardo, E. 1996, in The Cretaceous-Tertiary Event and Other catastrophes in Earth History, ed. G. Ryder, D. Fastovsky, \& S. Gartner (Boulder, CO: Geological Society of America), 105

Warren, P. H., Jerde, E. A., \& Kallemeyn, G. W. 1989, Earth Planet. Sci. Lett., 91,245

Warren, P. H., Kallemeyn, G. W., \& Kyte, F. T. 1999, Geochim. Cosmochim. Acta, 63, 2105

Weidenschilling, S. J. 2011, Icarus, 214, 671

Weidenschilling, S. J., Spaute, D., Davis, D. R., Marzari, F., \& Ohtsuki, K. 1997, Icarus, 128, 429 